\title{
Observation et modélisation de la structure verticale des vitesses et des concentrations de sédiments en suspension dans la zone de déferlement.
}

\author{
David Morellato $^{1,2}$ et Georges Chapalain ${ }^{1,2}$ \\ ${ }^{1}$ CETMEF \\ Technopôle Brest-Iroise - BP 5 - 29280 Plouzané, France \\ David.Morellato@developpement-durable.gouv.fr, \\ Georges.Chapalain@developpement-durable.gouv.fr \\ ${ }^{2}$ IUEM
}

Technopôle Brest-Iroise - Place Nicolas Copernic - 29280 Plouzané, France David.Morellato@univ-brest.fr, Georges.Chapalain@univ-brest.fr

\section{Résumé :}

Lors de campagnes réalisées en octobre 2006 et avril 2007 sur la plage de Pentrez, en baie de Douarnenez, nous avons cherché à quantifier la structure verticale des vitesses et des concentrations de sédiments en suspension dans la zone de déferlement. Ces mesures sont analysées et exploitées dans une démarche de modélisation numérique fondée sur les équations complètement non linéaires de WEI et al. (1995) (modèle FUNWAVE (KIRBY et al., 1998)) couplées à deux modèles de transport. Le premier est un modèle de transport global basé sur la formule de BAILARD (1981), le deuxième est un modèle vertical à fermeture turbulente. Le but de l'article est de déterminer la «meilleure » approche pour quantifier rapidement l'évolution des plages sableuses soumises aux vagues et à la marée.

\begin{abstract}
:
During campaigns (October, 2006 and April, 2007) on the beach of Pentrez, in the bay of Douarnenez, we tried to quantify the vertical structure of velocities and concentrations of suspended sediments in the breaking zone. These measures are analyzed and exploited in a numerical modelling approach based on the fully nonlinear equations of WEI et al. (1995) (model FUNWAVE (KIRBY et al., 1998), combined with two transport models. The first one is a global transport model based on the BAILARD (1981)'s formula, the second is a turbulent-closure vertical model. The purpose of the article is to determine the "best" approach to quantify quickly the evolution of sandy beaches subjected to waves and tide.
\end{abstract}




\section{Mots-clés :}

Houle, plage sableuse, équations de BOUSSINESQ, modèle numérique, morphodynamique, mesure, transport littoral.

\section{$1 \quad$ Introduction}

En ingénierie côtière, il est important de pouvoir quantifier le transport littoral, en vue d'appréhender l'évolution du trait de côte et par la suite d'élaborer des stratégies d'aménagement, comme le rechargement artificiel en sable fossile. Cette quantification peut être obtenue par l'utilisation de modèles numériques déterministes. Cependant, afin de pouvoir les utiliser, il est nécessaire de les caler sur des mesures hydrodynamiques et hydrosédimentaires, effectuées notamment dans la zone de déferlement, là où le transport littoral est prépondérant. En vue d'analyser l'impact de la houle et du marnage sur la dynamique littorale, un code de BOUSSINESQ a été implémenté et étendu au calcul des processus hydrosédimentaires, puis à l'évolution morphologique de l'avant-plage et de la plage via la résolution de l'équation de conservation du sédiment. La présente note est consacrée à la deuxième étape de ce travail qui consiste à mettre en œuvre deux modèles de transport de natures différentes et à confronter leurs résultats avec les mesures recueillies en baie de Douarnenez.

\section{Observations \\ 2.1 Site d'étude}

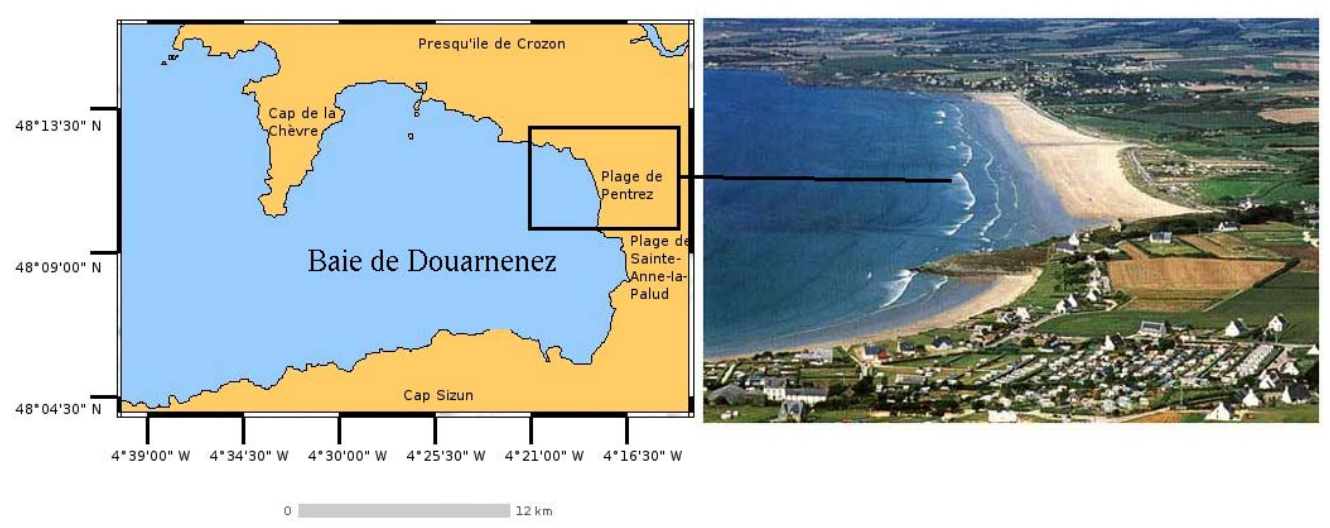

Figure 1. Localisation de la plage de Pentrez.

La plage de Pentrez, au fond de la baie de Douarnenez (voir Figure 1), est le site retenu. Cette plage de sable fin $\left(\mathrm{d}_{50}=140 \mu \mathrm{m}\right)$ est soumise à de faibles courants de marée (inférieurs à $5 \mathrm{~cm} / \mathrm{s}$ en condition de vive-eau) et à de fortes houles océaniques qui constituent l'agent hydrodynamique prédominant. Elle présente 
une pente constante de 1 à $2 \%$ et peu de variabilité longitudinale sur ses deux kilomètres de linéaire. Il s'agit d'une plage de type « dissipatif» avec des déferlements de type « glissant ».

\subsection{Mesures effectuées}

Pour mesurer la structure verticale des vitesses et des concentrations de sédiments en suspension aux environs du déferlement, nous avons conçu le MII (Mât Instrumenté en zone Intertidale), qui est un assemblage en inox ancré sur la plage (voir Figure 2). Sur cet assemblage, trois ou quatre ADV VECTOR, munis d'un OBS, sont fixés à différents niveaux $(30,60,90$ et $120 \mathrm{~cm})$, ce qui permet d'obtenir plusieurs points de mesure sur la verticale.

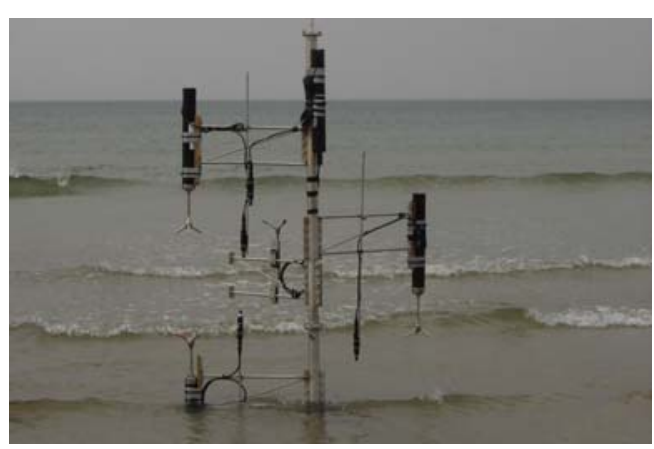

Figure 2. MII.
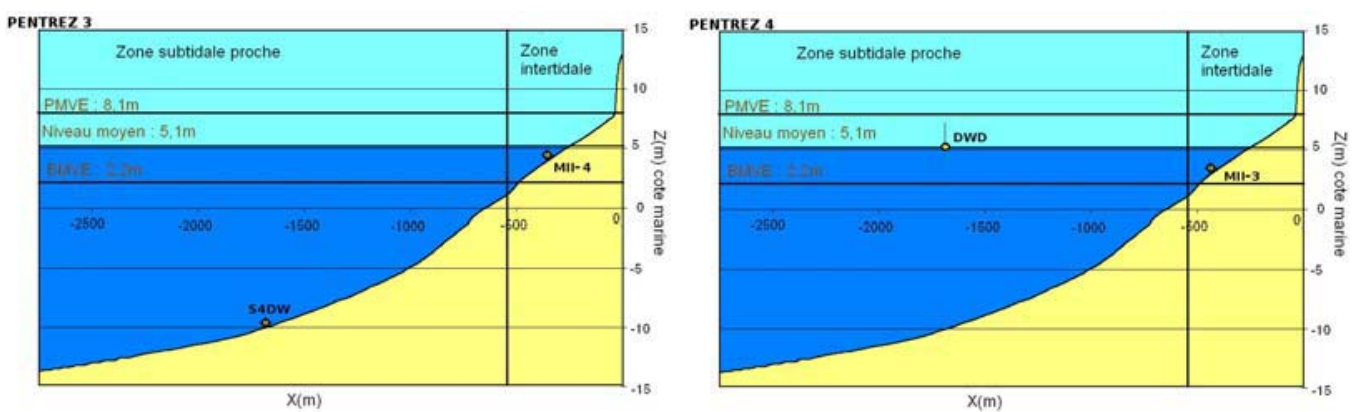

Figure 3. Localisation des instruments.

Nous avons effectué deux campagnes de mesures, PENTREZ 3 du 18 au 20 octobre 2006 et PENTREZ 4 du 14 au 16 avril 2007, au cours desquelles nous avons déployé le MII en zone intertidale. Pour compléter le dispositif, nous avons mouillé par $10 \mathrm{~m}$ de fond un courantomètre S4DW pour PENTREZ 3 et un houlographe DWD pour PENTREZ 4. La localisation des instruments est présentée sur la Figure 3. Tous les instruments ont permis d'acquérir en continu à la cadence de $1,28 \mathrm{~Hz}$ pour le houlographe, de $2 \mathrm{~Hz}$ pour le $\mathrm{S} 4 \mathrm{DW}$ et de $8 \mathrm{~Hz}$ pour les ADV VECTOR. 

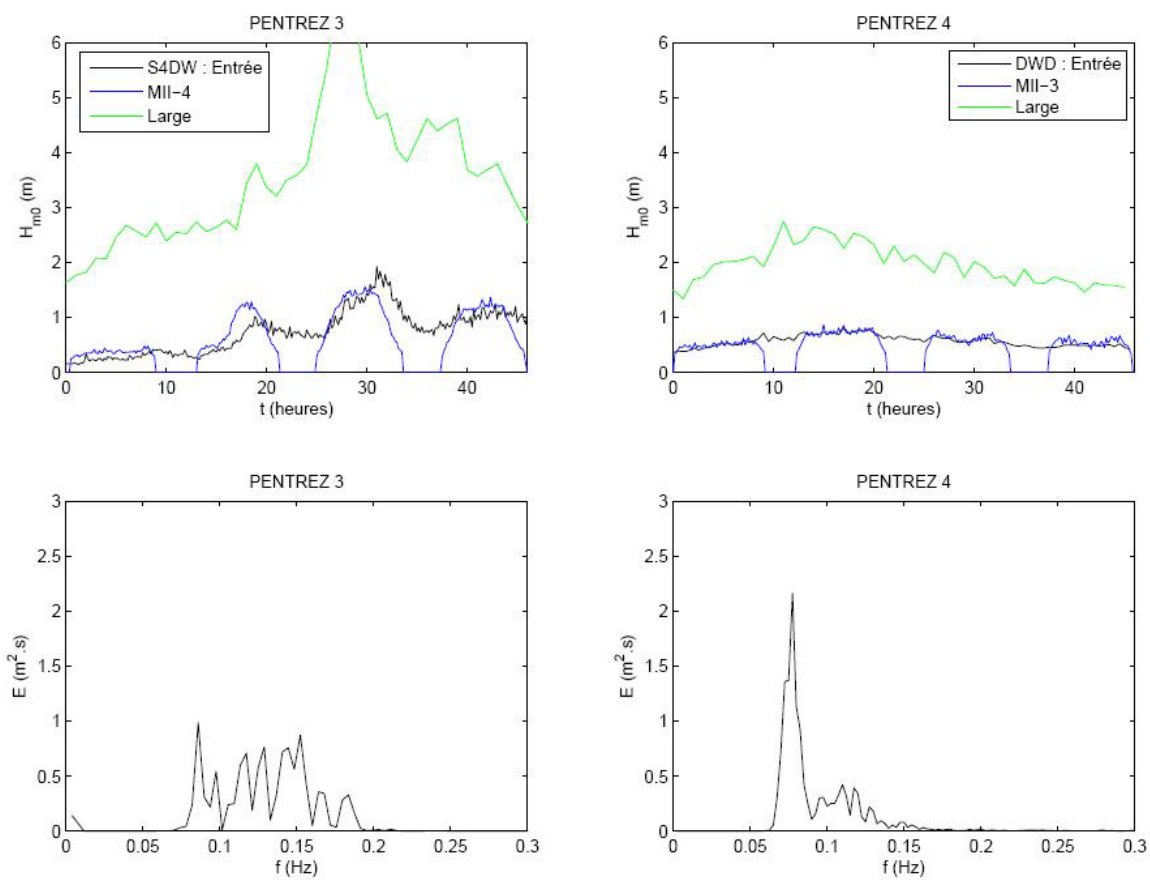

Figure 4. Hauteur significative spectrale des vagues et spectres correspondants.

La Figure 4 fait état de la chronique des vagues, observée pendant ces campagnes, et les spectres correspondants, représentatifs de l'état de mer, pour des hauteurs de vagues équivalentes (ici $0,81 \mathrm{~m}$ ), en entrée de profil. Elle montre la hauteur des vagues en entrée de profil (vers $-10 \mathrm{~m}$ ), au niveau du MII et au large, mesurée à une bouée située vers l'île d'Ouessant (Source : CETMEF). La campagne PENTREZ 3 a été marquée par une forte tempête, associée à un vent violent. Cela s'est caractérisé par la superposition d'une houle avec une forte mer de vent qui a généré des vagues jusqu'à $2 \mathrm{~m}$ au niveau du déferlement. La campagne PENTREZ 4 est caractérisée par une houle de beau temps quasimonochromatique atteignant au maximum $90 \mathrm{~cm}$ au niveau du déferlement.

\section{$3 \quad$ Modèle}

La modélisation consiste en un couplage entre un modèle de type BOUSSINESQ unidimensionnel (1D) et un modèle de transport. Nous avons choisi de tester un modèle de transport global et un modèle de transport basé sur un modèle vertical. Le modèle de BOUSSINESQ est appliqué le long du profil de plage instrumenté, de la position $\mathrm{z}=-10 \mathrm{~m}$ jusqu'au rivage; la chronique du niveau d'eau enregistrée par la bouée ou par le S4DW (après traitement) étant directement « injectée » comme condition à la limite extérieure du domaine de calcul. 


\subsection{Modèle de BOUSSINESQ}

Le modèle de BOUSSINESQ retenu est le modèle FUNWAVE (KIRBY et al., 1998). C'est un modèle numérique aux différences finies qui décrit la surface libre $\eta$ et la vitesse du courant $\mathrm{u}_{\alpha}$ à la profondeur $\mathrm{z}_{\alpha}=0,531 \mathrm{~h}$ au cours du temps $\mathrm{t}$ sur la base des équations complètement non linéaires de WEI et al. (1995) :

$$
\begin{array}{r}
\eta_{t}+M_{x}=0 \\
u_{\alpha t}+u_{\alpha} u_{\alpha x}+g \eta_{x}+V=0
\end{array}
$$

Avec $\mathrm{M}$ et les termes dispersifs $\mathrm{V}$ :

$$
\begin{aligned}
M & =(h+\eta)\left[u_{\alpha}+\left(z_{\alpha}+\frac{1}{2}(h-\eta)\right)\left(h u_{\alpha}\right)_{x x}\right. \\
& \left.+\left(\frac{1}{2} z_{\alpha}^{2}-\frac{1}{6}\left(h^{2}-h \eta+\eta^{2}\right)\right) u_{\alpha x x}\right] \\
V & =z_{\alpha}\left\{\frac{1}{2} z_{\alpha} u_{\alpha t x x}+\left(h u_{\alpha t}\right)_{x x}\right\}+\left\{\frac{1}{2}\left(z_{\alpha}^{2}-\eta^{2}\right) u_{\alpha} u_{\alpha x x}+\frac{1}{2}\left[\left(h u_{\alpha}\right)_{x}+\eta u_{\alpha x}\right]^{2}\right\}_{x} \\
& +\left\{\left(z_{\alpha}-\eta\right) u_{\alpha}\left(h u_{\alpha}\right)_{x x}-\eta\left[\frac{1}{2} \eta u_{\alpha t x}+\left(h u_{\alpha t}\right)_{x}\right]\right\}_{x}
\end{aligned}
$$

où $\mathrm{x}$ est l'abscisse, h la profondeur d'eau et $\mathrm{g}$ l'accélération de la pesanteur.

Ces équations décrivent l'évolution sans frottements de vagues non déferlantes, sur un fond imperméable et lisse. D'autres termes sont ajoutés dans ces équations pour tenir compte du déferlement, de la génération des vagues et du frottement sur le fond. Le frottement est évalué grâce au prédicteur de rides de NIELSEN (1992) qui permet de calculer le coefficient de frottement $f_{w}(x)$.

La structure verticale de la vitesse est donnée par :

$$
u_{\mathrm{FUNWAVE}}(z)=u_{\alpha}+\frac{z_{\alpha}^{2}-z^{2}}{2} u_{\alpha x x}+\left(z_{\alpha}-z\right)\left(h u_{\alpha}\right)_{x x}
$$

Au niveau du déferlement, l'excès d'eau apporté par la vague crée un courant au fond dirigé vers le large, non reproduit par ce modèle. Les modèles de transport reproduisent ce courant pour estimer de façon correcte le transport.

\subsection{Modèle de transport global}

Pour palier à ce problème, LYNETT (2006) a mis en place un post-traitement pour tenir compte du courant de retour induit par le déferlement. Il modifie la vitesse verticale par la relation suivante :

$$
u_{\text {LYNETT }}(z)=u_{\mathrm{FUNWAVE}}(z)+u_{B}(z)
$$


avec

$u_{B}(z)=\delta_{\text {déferlement }}\left(C-u_{\mathrm{FUNWAVE}}(\eta)\right) \frac{\exp (k(z-\eta))-\exp \left(k\left(z_{B}-\eta\right)\right)}{1-\exp \left(k\left(z_{B}-\eta\right)\right)}$

où $\mathrm{C}$ est la vitesse du rouleau, $\mathrm{k}$ et $\mathrm{z}_{\mathrm{B}}$ des constantes. L'expression de cette vitesse instantanée au niveau du fond $\mathrm{u}(\mathrm{x},-\mathrm{h}, \mathrm{t})$ est utilisée dans la formule de BAILARD (1981) pour évaluer le transport. Cette formule permet ainsi de distinguer le transport en suspension $\mathrm{q}_{\mathrm{s}}$, le charriage $\mathrm{q}_{\mathrm{c}}$ et l'effet de la pente $\beta_{\mathrm{p}}(\mathrm{x})\left(\mathrm{q}_{\mathrm{s} \beta}+\mathrm{q}_{\mathrm{c} \beta}\right)$ :

$$
q_{1 \mathrm{D}}(x)=q_{c 0}(x)+q_{c \beta}(x)+q_{s 0}(x)+q_{s \beta}(x)
$$

avec

$$
\begin{aligned}
q_{c 0}(x) & =\frac{f_{w}(x) \varepsilon_{c}}{2 g(s-1) \tan \phi} \overline{|u(x,-h, t)|^{2} u(x,-h, t)} \\
q_{c \beta_{p}}(x) & =-\frac{f_{w}(x) \varepsilon_{c}}{2 g(s-1) \tan ^{2} \phi} \overline{|u(x,-h, t)|^{3}} \beta_{p}(x) \\
q_{s 0}(x) & =\frac{f_{w}(x) \varepsilon_{s}}{2 g(s-1) w_{f}} \overline{|u(x,-h, t)|^{3}} \\
q_{s \beta_{p}}(x) & =-\frac{f_{w}(x) \varepsilon_{s}^{2}}{2 g(s-1) w_{f}^{2}} \overline{|u(x,-h, t)|^{5}} \beta_{p}(x)
\end{aligned}
$$

où $\varepsilon_{\mathrm{c}}=0,1$ et $\varepsilon_{\mathrm{s}}=0,02$ sont les facteurs d'efficacité du transport par charriage et en suspension, $\mathrm{w}_{\mathrm{f}}$ la vitesse de chute, $\mathrm{s}$ la densité et $\varphi$ l'angle de frottement $\mathrm{du}$ sédiment. Le couplage du modèle FUNWAVE avec ce modèle de transport nous fournit un modèle $1 \mathrm{D}$ horizontal.

\subsection{Modèle vertical}

En tous points $\mathrm{x}$ où est évalué le transport, nous avons mis en place un modèle vertical en coordonnées $\sigma$, basé sur l'équation d'énergie cinétique turbulente, inspiré du modèle développé par DEIGAARD et al. (1991). Les équations du modèle permettant d'obtenir la vitesse instantanée $u(z, t)$ et la concentration instantanée $\mathrm{C}(\mathrm{z}, \mathrm{t})$ sont :

$$
\begin{aligned}
\frac{\partial u}{\partial t}-\frac{\sigma}{H} \frac{\partial \eta}{\partial t} \frac{\partial u}{\partial \sigma} & =\frac{\partial \bar{u}_{0}}{\partial t}+\frac{\partial}{\partial \sigma}\left(\nu_{t} \frac{\partial u}{\partial \sigma}\right) \frac{1}{H^{2}}-\frac{1}{\rho_{w}} \frac{\partial \bar{\tau}_{z x}}{\partial z} \\
\frac{\partial k}{\partial t}-\frac{\sigma}{H} \frac{\partial \eta}{\partial t} \frac{\partial k}{\partial \sigma} & =\frac{1}{H^{2}} \frac{\partial}{\partial \sigma}\left(\frac{\nu_{t}}{\sigma_{k}} \frac{\partial k}{\partial \sigma}\right)+\frac{\nu_{t}}{H^{2}}\left(\frac{\partial u}{\partial \sigma}\right)^{2}-C_{\nu} \frac{k^{\frac{3}{2}}}{l} \\
\frac{\partial C}{\partial t}-\frac{\sigma}{H} \frac{\partial \eta}{\partial t} \frac{\partial C}{\partial \sigma} & =\frac{1}{H} \frac{\partial}{\partial \sigma}\left[\left(w_{f}-w_{\sigma}\right) C\right]+\frac{1}{H^{2}} \frac{\partial}{\partial z}\left(\varepsilon_{s d} \frac{\partial C}{\partial z}\right)
\end{aligned}
$$


où $\mathrm{H}=\mathrm{h}+\eta$ est la hauteur d'eau instantanée, $\mathrm{k}$ l'énergie cinétique turbulente, 1 la longueur de mélange, $v_{\mathrm{t}}=\varepsilon_{\mathrm{sd}}$ le coefficient de viscosité turbulente égal au coefficient de diffusion turbulente, $\mathrm{w}_{\sigma}$ la vitesse verticale, $\mathrm{u}_{0}$ la vitesse orbitale des vagues, $\bar{\tau}_{\mathrm{zx}}$ la contrainte moyenne sur une période de vagues et $\sigma_{\mathrm{k}}=1, \mathrm{C}_{\mathrm{v}}=0,08$ des constantes. Grâce au couplage entre le modèle vertical et la formule de charriage, nous obtenons le taux de transport total :

$$
q_{2 \mathrm{DV}}(x)=\bar{q}_{s u}(x)+\bar{q}_{s w}(x)+\bar{q}_{s l}(x)+\bar{q}_{c}(x)
$$

avec

$$
\begin{aligned}
\bar{q}_{s u} & =\overline{\int_{z_{0}}^{H} C(z) \bar{u}(z) \mathrm{d} z} \\
\bar{q}_{s w} & =\frac{\int_{z_{0}}^{H} C(z) u_{w}(z, t) \mathrm{d} z}{\int_{z_{0}}^{H} C(z) U_{l}(z) \mathrm{d} z} \\
\bar{q}_{s l} & =q_{c 0}+q_{c \beta}
\end{aligned}
$$

où $\mathrm{u}=\mathrm{u}_{\mathrm{w}}+\overline{\mathrm{u}}$ et $\mathrm{U}_{1}$ est la vitesse Lagrangienne, contribution nécessaire quand on néglige les termes convectifs horizontaux dans le calcul des concentrations de sédiments (BRØKER et al., 1991, RAKHA et al., 1997). Le couplage du modèle FUNWAVE avec le modèle de transport vertical nous fournit un modèle 2DV.

Un calcul pour le modèle de houle et pour les deux modèles de transport est effectué toutes les 10 minutes (pas morphodynamique $\Delta \mathrm{t}$ ) où les pas spatial et temporel valent respectivement $1 \mathrm{~m}$ et $0,065 \mathrm{~s}$.

\section{$4 \quad$ Résultats et discussion}

La Figure 5 montre la hauteur de houle significative spectrale $\mathrm{H}_{\mathrm{m} 0}$ issue de la modélisation avec les mesures. Le meilleur accord est trouvé lors de la campagne PENTREZ 4.

La Figure 6 et la Figure 7 présentent les vitesses moyennes prédites et mesurées. Pour les vitesses prédites, nous remarquons peu d'écart entre le modèle de LYNETT (2006) et le modèle vertical, ainsi qu'une très bonne corrélation avec les mesures auxquelles nous avons pris le soin de soustraire la composante moyenne liée au courant de marée. Nous remarquons que la vitesse moyenne est maximale et est dirigée vers le large au moment du déferlement. 

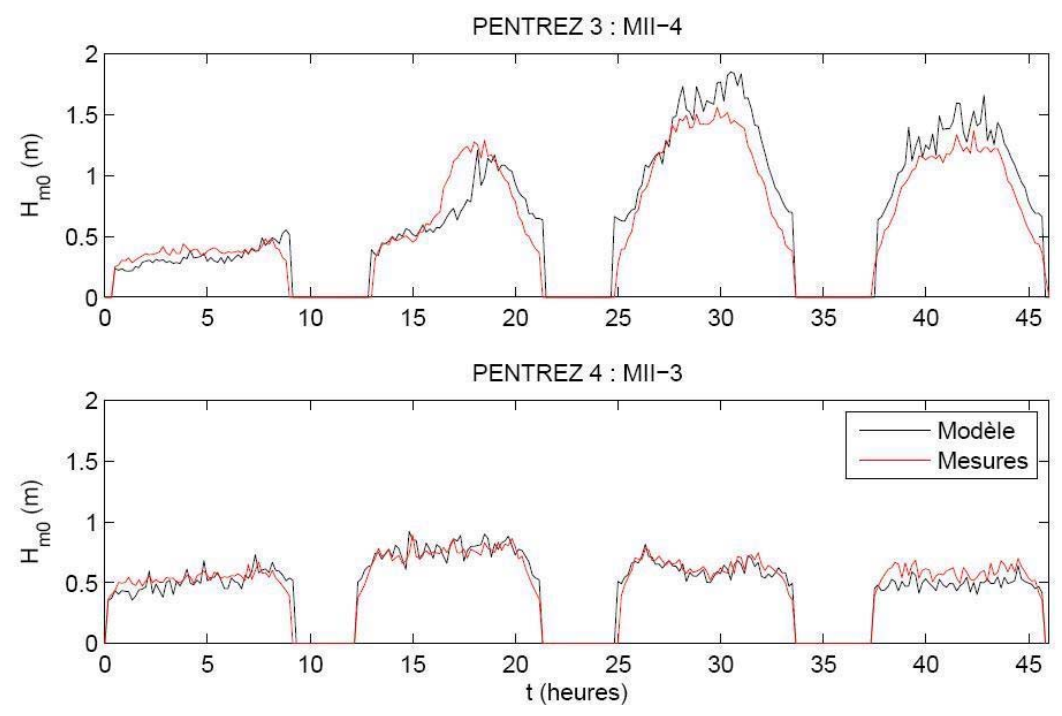

Figure 5. Hauteur significative spectrale des vagues prédite et mesurée aux points MII.

La Figure 8 et la Figure 9 comparent les concentrations de sédiments en suspension (CSS) mesurées avec le modèle vertical. Les résultats sont assez satisfaisants après calage des concentrations de référence. Nous remarquons que la CSS est maximale au moment du déferlement.

La Figure 10 montre les taux de transport issus du modèle de transport global 1D (valeurs par défaut) et du modèle de transport 2DV aux points de mesure. Nous observons quelques différences de comportement entre les deux modèles mais les ordres de grandeur sont respectés, ce qui montre que le modèle de transport $1 \mathrm{D}$ est calé sur le modèle de transport 2DV.

Des simulations effectuées sur plusieurs points (MORELLATO, en cours) montrent que le signal du taux de transport est assez bruité, ce qui entraîne des instabilités au niveau du calcul morphodynamique. Sur le calculateur CAPARMOR (IFREMER), pour un $\Delta \mathrm{t}$ de 10 minutes, il faut quelques secondes pour évaluer le transport avec le modèle $1 \mathrm{D}$ sur tout le domaine et 20 minutes avec le modèle 2DV en un seul point. Les résultats n'étant pas meilleurs avec un modèle vertical et étant plus difficiles à obtenir, nous préférons pour la suite utiliser le modèle de transport global dans nos prévisions morphodynamiques. 
PENTREZ 3: MII-4
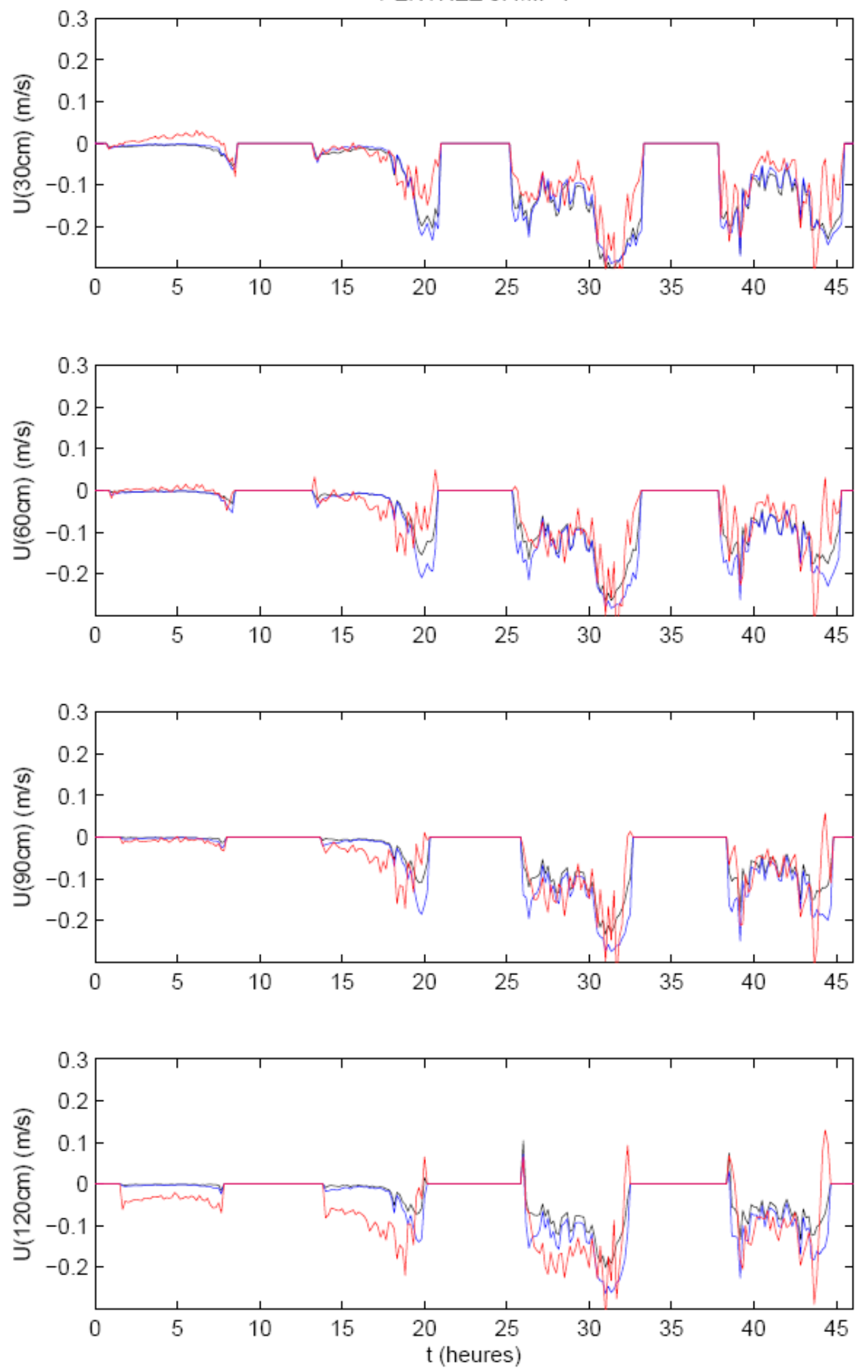

Figure 6.Vitesses moyennes prédites et mesurées sur PENTREZ 3. 

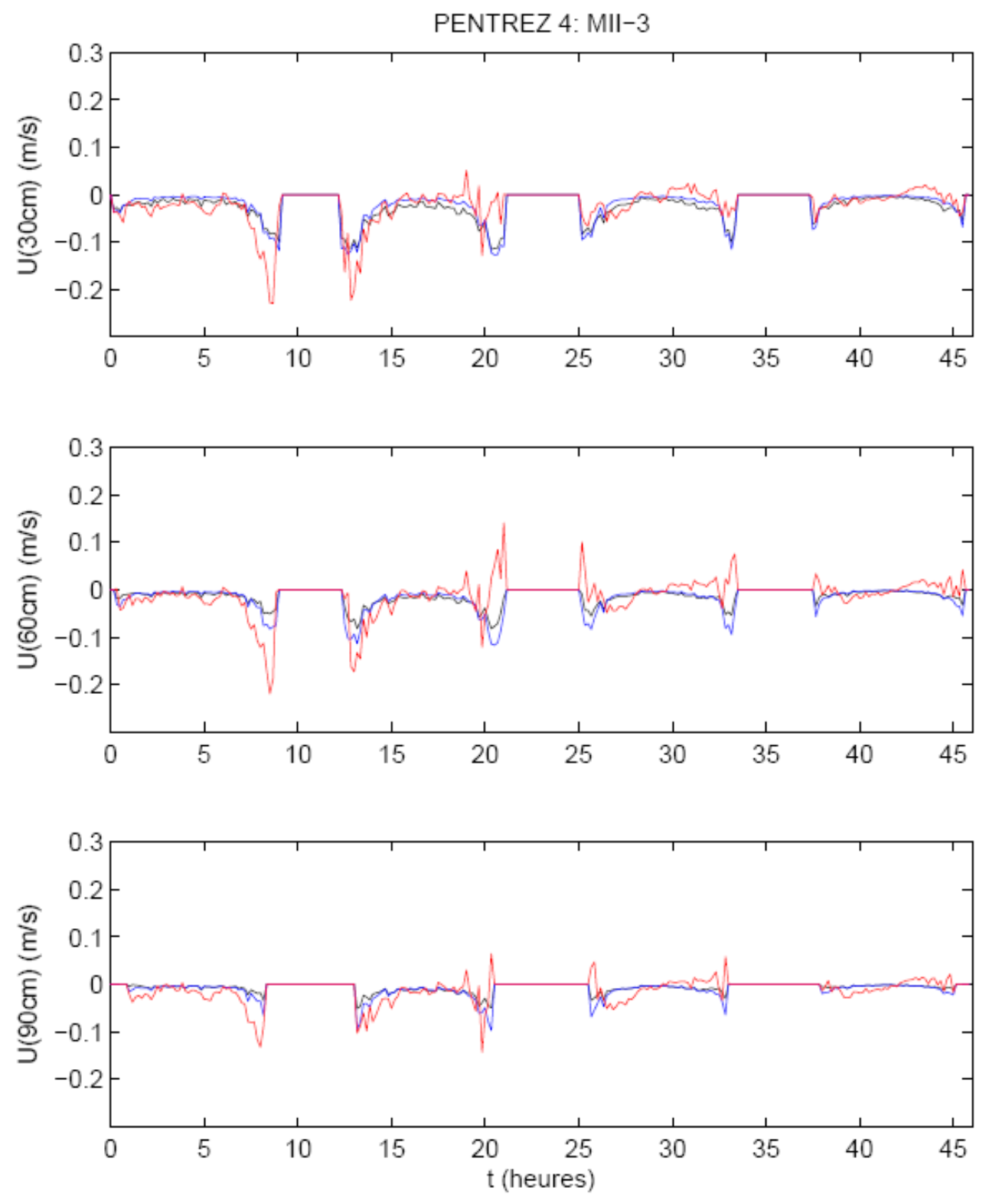

Figure 7.Vitesses moyennes prédites et mesurées sur PENTREZ 4. 

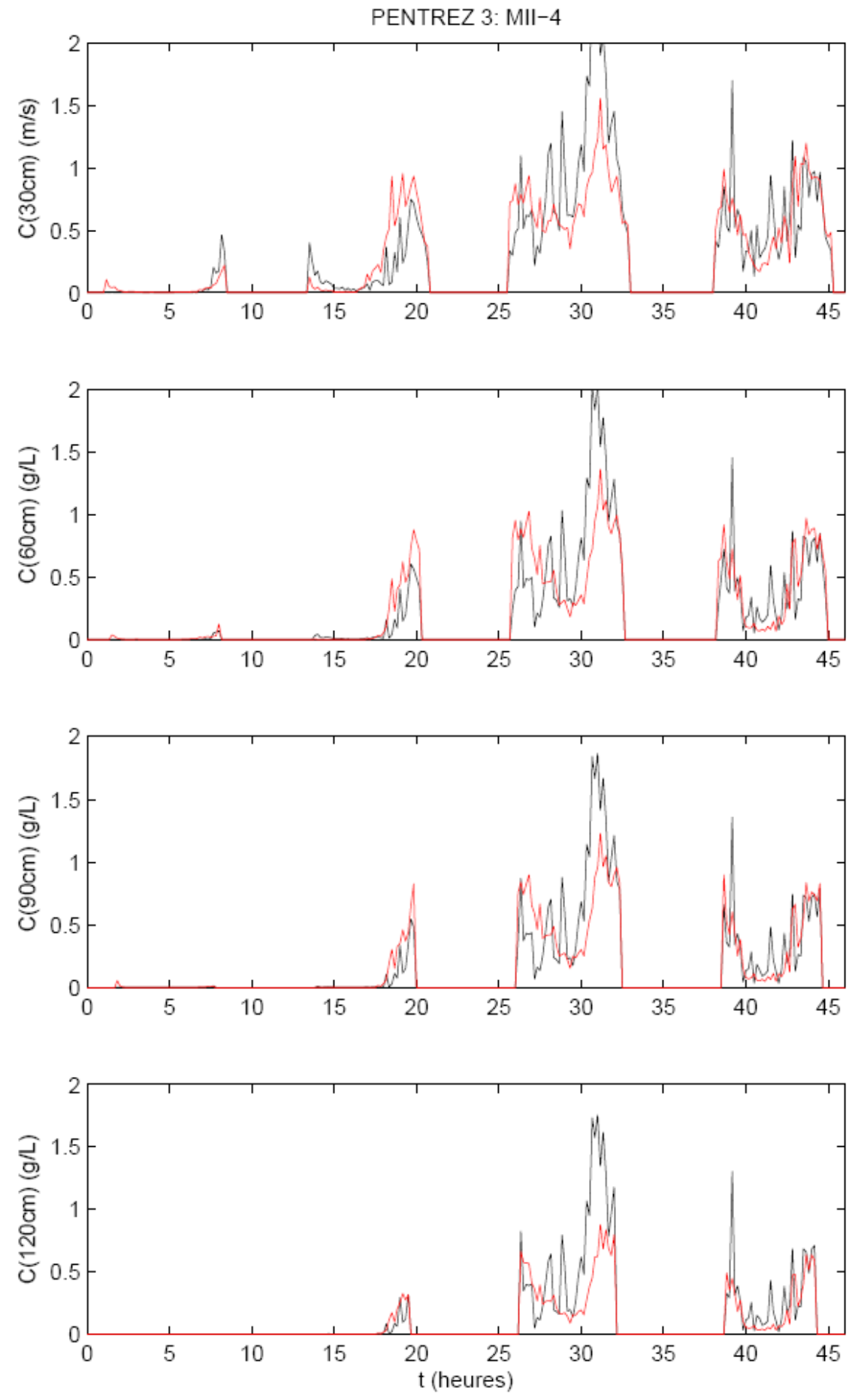

Figure 8.CSS moyennes prédites et mesurées sur PENTREZ 3. 

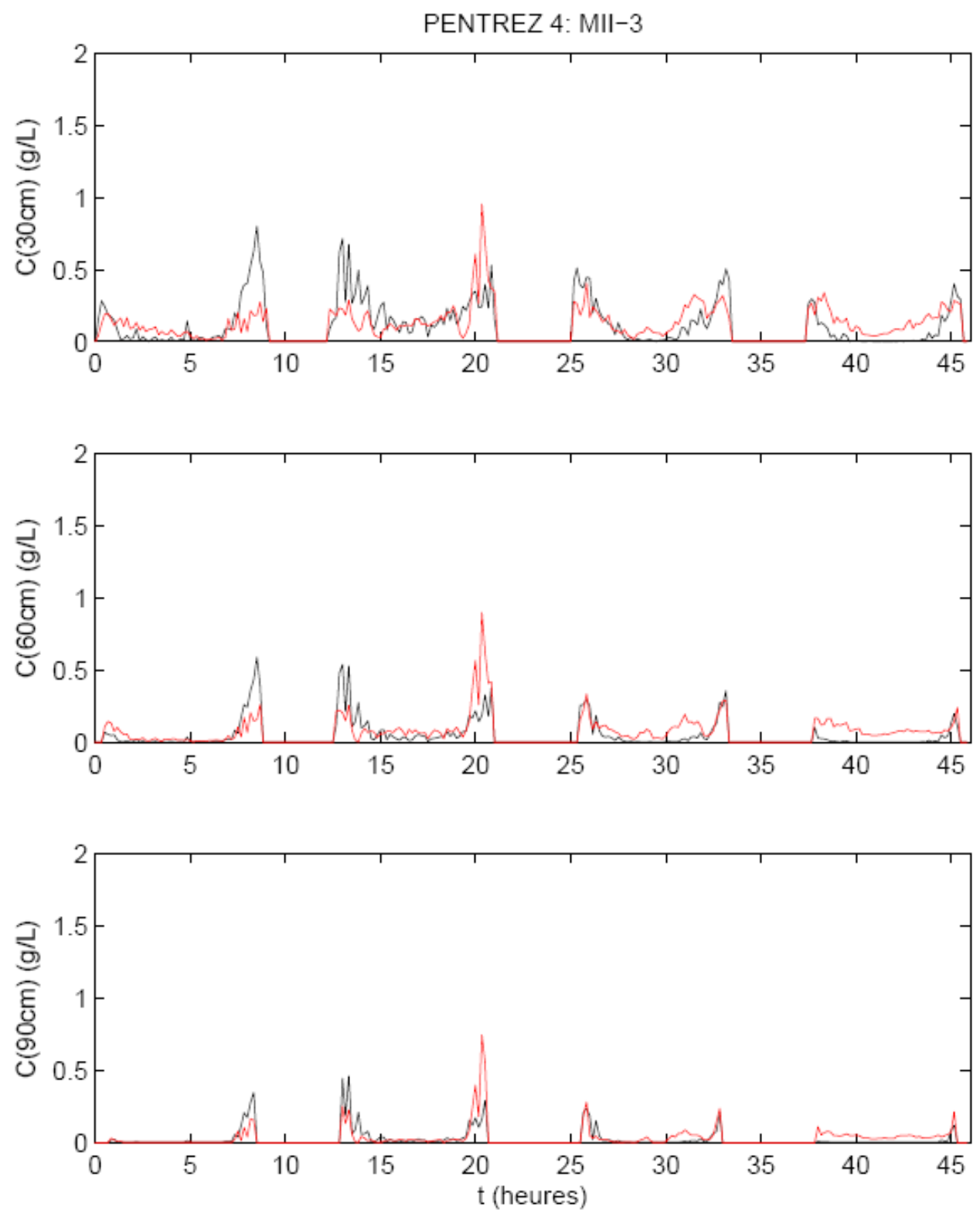

Figure 9.CSS moyennes prédites et mesurées sur PENTREZ 4. 

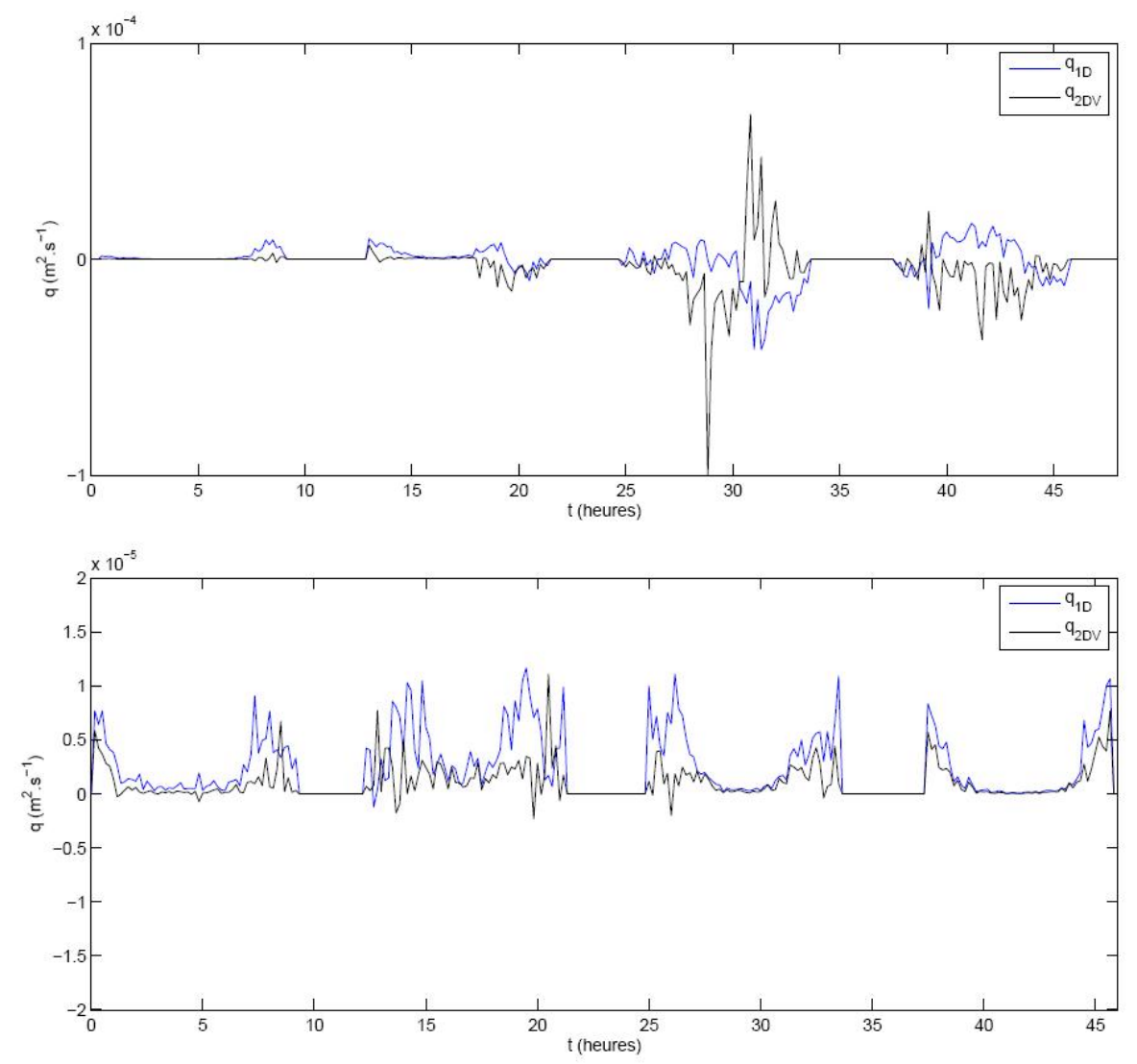

Figure 10. Taux de transport modélisés aux points de mesures (En haut, PENTREZ 3 ; en bas, PENTREZ 4).

\section{Conclusion}

La présente étude montre qu'un modèle vertical n'est pas utile pour faire des prévisions morphodynamiques. Son intérêt réside dans le calage de formules de transport sur les résultats de ce modèle. Une description analytique pour calculer la vitesse au fond, couplée à un modèle de transport énergétique, suffit pour obtenir des résultats corrects et rapides. A l'issue de ce travail, nous avons développé un modèle morphodynamique fonctionnel et calé sur la plage de Pentrez.

\section{$6 \quad$ Remerciements}

Nous adressons nos vifs remerciements au personnel du CETMEF (G. Amis, N. Guillou, A. Le Berre et A. Le Flem), du LEMAR (UMR CNRS-UBO 6539 LEMAR) (E. Amice, L. Chauvaud, E. Duvieilbourg et R. Marc) et de la commune de Saint-Nic, pour leur contribution à l'acquisition des mesures de terrain pendant les campagnes PENTREZ 3 et PENTREZ 4. 


\section{$7 \quad$ Références bibliographiques}

1 BAILARD J. A. (1981). An energetics total load sediment transport model for a plane sloping beach. J. Geophys. Res, 86, pp10938-10954.

2 BRØKER I., DEIGAARD R. et FREDSØE J. (1991). On/offshore sediment transport and morphological modelling of coastal profiles. ASCE Specialty conf. Coastal Sediments'91, Seattle, WA, pp 643-653.

3 DEIGAARD R., JUSTESEN P. et FREDSØE J. (1991). Modeling of undertow by one-equation turbulence model. Coastal Engineering, 15, pp 431-458.

4 KIRBY J. T., WEI G., CHEN A., KENNEDY A. B. et DALRYMPLE, R. A. (1998). FUNWAVE 1.0 Fully nonlinear Boussinesq wave model. Documentation and user's manual. Rapport de recherche CACR-98-06, Center for Applied Coastal Research, Department of Civil and Environmental Engineering, University of Delaware.

5 LYNETT P. J. (2006). Wave breaking velocity effects in depthintegrated models. Coastal Engineering, 53(4), pp 325-333.

6 MORELLATO (en cours). Influence de la houle et du marnage sur la morphologie des plages sableuses. Thèse de Doctorat, Université de Bretagne Occidentale.

7 NIELSEN P.(1992). Coastal bottom boundary layers and sediment transport. Advanced Series on Ocean Engineering, world scientific.

8 RAKHA R., BRØKER I. et DEIGAARD R. (1997). A phase-resolving cross shore sediment transport model for beach profile evolution. Coastal Engineering, 31, pp 231-261.

9 WEI G., KIRBY J. T., GRILLI S. T. ET SUBRAMANYA, R. (1995). $A$ fully nonlinear Boussinesq model for surfaces waves. Part1. Highly nonlinear unsteady waves. J. Fluid Mech., 294, pp 71-92. 